\title{
Focusing on Risk Communication About Medicines Why Now?
}

\author{
Priya Bahri ${ }^{1}$ and Mira Harrison-Woolrych ${ }^{2}$ \\ 1 European Medicines Agency, London, UK \\ 2 Intensive Medicines Monitoring Programme, New Zealand Pharmacovigilance Centre, University of \\ Otago, Dunedin, New Zealand
}

Communication about the risks of medicines is not a new issue. Patients have long since sought advice about remedies, whether from family, healthcare workers or the wider community in traditional ways, or via websites and social media in today's world. As pharmacovigilance professionals, interacting with patients, healthcare professionals, health policy makers, journalists and the general public has become part of our daily activity, in order to manage risks with medicines appropriately and reduce patient harm.

So why is this theme edition of Drug Safety focusing on risk communication now? A decade ago, risk management was introduced into pharmacovigilance, ${ }^{[1]}$ and the implementation and evaluation of this approach are still subject to improvement in the light of experience gained so far. But it has become clear that at the core of successful risk management lies effective risk communication. This applies to formal risk management plans as well as to risk minimization action otherwise taken for a medicine and interactions between healthcare professionals and patients. Drug Safety has increasingly been a forum for discussing risk communication, ${ }^{[2-11]}$ and given that applying communication science to pharmacovigilance is relatively new, we thank the journal editors for now dedicating a whole edition to this topic.

Articles were invited from authors with different kinds of relevant expertise and with a view to covering perspectives from various stakeholders and world regions. The resulting contributions include original research, reviews and opinion papers. It is perhaps not surprising - due to the still limited research - that often opinions are underpinned by experiences rather than systematic evidence, and this illustrates the need for further research. There is now an urgent need to look at old and new ideas for risk communication under a magnifying glass, in order to explore how to put established principles into practice and to experiment with new approaches.

Why is this urgent? Avoidable adverse reactions and medication errors are still a leading cause for morbidity and mortality, ${ }^{[12-21]}$ and there are ongoing patient needs for information to facilitate treatment choices. ${ }^{[22]}$

In their article on patients' expectations, Cox and Butt ${ }^{[23]}$ remind us of the need for information about benefits as well as risks and that prescribers may prefer to highlight benefits rather than risks of medicines. Referring to their own research, they stress that patients often lack understandable risk information and want their negative experiences to be taken seriously. This is described as fundamental for the trust of patients in their healthcare professionals and treatments. Furthermore, the authors discuss that differences in risk perceptions between patients and healthcare professionals may be a barrier to one-to-one communication. 
This barrier also exists when communicating with broader audiences, and Dodoo and Hugman ${ }^{[24]}$ give us important advice in this respect: public concerns have to be addressed, whether or not, as pharmacovigilance professionals, we share these concerns. The authors take us on a journey through sub-Saharan Africa, with examples illustrating how risk communication may be linked with social problems and citizens' trust - or often mistrust - in governments. We should hence understand that we cannot look at a safety concern in isolation, but that the social and political context should be considered for every communication intervention about a medicine with public health relevance. Every time communication fails, public trust in medicines is eroded and this trust is difficult to re-build. This may result in using medicines inappropriately or avoiding them altogether, which could threaten patient and public health. The need to establish trust in pharmacovigilance systems at both individual and population level adds to the urgency of this theme edition.

Similar lessons can be learnt from the Philippines, and Hartigan- $\mathrm{Go}^{[25]}$ advises us to keep ourselves on a constant learning curve, as past experiences should inform future communication strategies. He advocates that risk management and communication should become an accepted culture within governments, healthcare and pharmaceutical industry, and recommends applying the social marketing approach for promoting safe use of medicines. Among the many challenges he describes as prevalent in the developing world is also the difficulty for healthcare professionals to keep aware of all latest evidence.

This is not easy elsewhere either, as reported from the Netherlands by Piening et al. ${ }^{[26]}$ By means of a survey, they further found that the majority of Dutch healthcare professionals considered receiving risk communication important and that almost one-third reported taking action following a direct healthcare professional communication (DHPC). This might be considered a disappointing figure, but in some other areas achieving a behavioural impact by a single act of communication in one-third of the targeted audience would be a success. The authors suggest a mix of tools for disseminating information to impact on more healthcare professionals, as necessary for patient safety. This kind of research into healthcare professional and other audiences is much needed and we hope to see more in other countries too. We will also need objective measurements of behaviour, rather than relying on self-reported impact. The impact crucially depends on the trust in the information source, and Piening et al. ${ }^{[26]}$ found that healthcare professionals prefer to be informed by governmental or their own professional bodies, and not by pharmaceutical industry.

Edwards and Chakraborty ${ }^{[27]}$ discuss communication from manufacturers, including public mistrust. They highlight that improvements through proper effectiveness evaluation of communication remains to be achieved and suggest learning from patient information leaflets, which have been improved much through testing. For manufacturers' communication strategies, the authors recommend coherent strategies across the organization and agreeing the strategy's objectives with the regulatory authority. But they also conclude that common understanding between manufacturers and regulators is often lacking, as to whether risk communication intends to inform, to help understanding or to change behaviour of medicine users. So working together towards a common understanding is fundamental for future progress.

How regulators can gain clarity on their communication objectives can be learnt from the article by Fischhoff. ${ }^{[28]} \mathrm{He}$ proposes a framework for decision making on the benefit-risk balance of medicines, which both requires and facilitates communication. The framework integrates calculations and statistics with judgement on the quality and strength of the evidence, based on transparently agreed values and criteria. Methodological clarity should facilitate communication between experts, decision makers and other stakeholders, with a view to explaining to the public why a certain decision was taken. This honesty about valuebased judgements upon the evidence may enable us, as pharmacovigilance experts, to communicate effectively in the given social and political contexts.

These contexts have been studied for vaccines more than for most other medicines, and Larson et al. ${ }^{[29]}$ give us proof of many points discussed in 
other articles in this theme edition, with major examples for vaccines. Again, there is advocacy for an integrated approach of risk assessment, minimization and communication, also integrating the monitoring of public concerns. The authors make other very important points: listening must be part of communication, and rather than sticking to standard messages that have not worked in certain circumstances, messages should be tailored to respect each community, address their specific concerns and gain their trust. This approach could increase vaccine acceptance, and given the examples of (re)occurrence of vaccine-preventable disease outbreaks, this is another urgent call.

While we may realize that we need to urgently improve risk communication, we should also understand that to make that happen a fundamental shift is needed in the way we work as pharmacovigilance professionals, in whichever setting.

To help us in making that shift, Hugman ${ }^{[30]}$ whirls us across countries, backwards and forwards in time and through fiction and non-fiction. While appreciating the principles of democratic public administrations to provide services to the public based on equality and fairness, he warns us of obstacles administrations can create with negative impact on communication. He applies his criticism of bureaucracies not only to public administrations, but also to those in healthcare and pharmaceutical industry. In order to overcome rigid mindsets, he draws our eyes to compassionate problem solving through 'creative bureaucracies' which are honest, ethical, efficient, motivating, non-hierarchical, networking, interdisciplinary, multicultural and, of course, patient-centred! Ultimately, Hugman ${ }^{[30]}$ gives us the lens for our focus in pharmacovigilance in general and communication in particular: how can we ensure that everything we do contributes to patient safety and welfare?

Applying this lens to the integration of risk assessment, minimization and communication and decision making upon the benefit-risk balance of a medicine, shows us that an integrating framework should not add procedural obstacles, but facilitate problem solving.

We also agree that this should result in communication interventions tailored for specific audiences and that 'not one size fits all', as discussed in articles in this theme edition. However, we face a dilemma when we have to communicate with broad audiences as regards how far we can tailor messages without appearing to issue inconsistent messages. Also, since communication needs and preferences differ by many factors, we should reflect upon the need for, but also the feasibility of, audience segment-specific data versus the generalizability of less segmented data.

Furthermore, we must consider that patients and healthcare professionals may already be confused by different information sources, as some authors highlight. Team effort, in particular with listening to patients and working with patient organizations, is therefore required from as many stakeholders as possible to get agreement on messages. Embracing our world of plurality, we need to strengthen our communication practices in order to make our voice heard in the interest of patients.

Where should we focus now in order to progress? Not all ideas presented in this theme edition are new - most importantly we should remember the Erice Report ${ }^{[31]}$ and its recent update enforcing patients' interests $^{[32]}$ - but implementation is not so easy. While progress has been achieved in some areas, other established principles of good communication practices are not yet systematically followed. Also, the communication environment has seen rapid changes recently in terms of social and political contexts, such as increased public demands for information, as well as significant developments in information technology and related communication behaviour. This theme edition reflects upon the challenges these changes present, and we are grateful to the authors for inspiring us with new ideas on how to tackle these.

Ultimately, the usefulness of all our efforts in pharmacovigilance will manifest itself in our ability to communicate practical advice for the safe use of medicines. This advice should achieve improved patient health outcomes. This is a high goal, but we cannot settle for less. Inadequate communication is a risk in itself, and hence communication expertise and functions within pharmacovigilance should be installed alongside all other crucial functions. Let's focus on this now - the safety of patients will depend on it. 


\section{Acknowledgements}

The views expressed in this editorial are the personal views of the guest editors and may not be understood or quoted as being made on behalf of or reflecting the position of any of the organizations the guest editors are affiliated to. No sources of funding were used to prepare this article and the guest editors have no conflicts of interest to declare.

This editorial is part of a theme issue co-edited by Priya Bahri, European Medicines Agency, UK, and Mira HarrisonWoolrych, New Zealand Pharmacovigilance Centre, New Zealand. No external funding was used to support the publication of this theme issue.

\section{References}

1. The future is now: current concepts in risk management [topical edition]. Drug Saf 2004; 27: 489-617

2. Edwards R, Hugman B. The challenge of effectively communicating risk-benefit information. Drug Saf 1997; 17: 216-27

3. Goldman SA. Communication of medical product risk: how effective is effective enough? Drug Saf 2004; 27: 519-34

4. Czarnecki A. Intelligent risk communication: can it be improved? Drug Saf 2008; 31: 1-6

5. Edwards IR, Lindquist M. Understanding and communicating key concepts in risk management: what do we mean by benefit and risk? Drug Saf 2009; 32: 449-52

6. Sanfélix-Gimeno G, Cervera-Casino P, Peiró S, et al. Effectiveness of safety warnings in atypical antipsychotic drugs: an interrupted time-series analysis in Spain. Drug Saf 2009; 32: 1075-87

7. Yong PL, Bigman C, Flynn DN, et al. Messages about black-box warnings: a comparative analysis of reports from the FDA and lay media in the US. Drug Saf 2009; 32: $1147-57$

8. Mol PGM, Straus SMJM, Piening S, et al. A decade of safety-related regulatory action in the Netherlands: a retrospective analysis of direct healthcare professional communications from 1999-2009. Drug Saf 2010; 33: 463-74

9. Bahri P. Public pharmacovigilance communication: a process calling for evidence-based, objective-driven strategies. Drug Saf 2010; 33: 1065-79

10. Bahri P, Mol PGM, Théophile H, et al. Communication in drug safety: a report from an interactive debate held at the 10th Annual Meeting of the International Society of Pharmacovigilance (ISoP), 2010. Drug Saf 2011; 34 $881-2$

11. Piening S, Haaijer-Ruskamp FM, de Vries JTN, et al. Impact of safety-related regulatory action on clinical review: a systematic review. Drug Saf 2012; 35: 373-85

12. Muehlberger N, Schneeweiss S, Hasford J. Adverse drug reaction monitoring: cost and benefit considerations. Part I: frequency of adverse drug reactions causing hospita admissions. Pharmacoepidemiol Drug Saf 1997; 6 Suppl. 3 : S71-7

13. Goettler M, Schneeweiss S, Hasford J. Adverse drug reaction monitoring: cost and benefit considerations. Part II cost and preventability of adverse drug reactions leading to hospital admission. Pharmacoepidemiol Drug Saf 1997; 6 Suppl. 3: S79-90

14. Pouyanne P, Haramburu F, Imbs JL, et al. Admissions to hospital caused by adverse drug reactions: cross-sectional incidence study. BMJ 2000; 320: 1036

15. Phillips J, Beam S, Brinker A, et al. Retrospective analysis of mortalities associated with medication errors. Am J Health System Pharm 2001; 58: 1835-41

16. Beijer HJM, Blaey de CJ. Hospitalisations caused by adverse reactions (ADR): a meta-analysis of observational studies. Pharm World Sci 2002; 24: 46-54

17. Institute of Medicine of the National Academies (IOM). Preventing medication errors. Washington, DC: National Academies Press, 2006

18. Howard RL, Avery AJ, Slavenburg S, et al. Which drugs cause preventable admissions to hospital? A systematic review. Br J Clin Pharmacol 2006; 63: 136-47

19. Council of Europe Expert Group on Safe Medication Practices. Creation of a better medication safety culture in Europe: building up safe medication practices. Strasbourg: Council of Europe, 2007

20. Leendertse AJ, Egberts ACG, Stoker LJ, et al., for the HARM study group. Frequency of and risk factors for preventable medication-related hospital admissions in the Netherlands. Arch Intern Med 2008; 168: 1890-6

21. Hakkarainen KM, Hedna K, Petzold M, et al. Percentage of patients with preventable adverse drug reactions and preventability of adverse drug reactions: a meta-analysis. PLoS One 2012; 7: e33236, 1-9

22. International Alliance of Patients' Organizations. IAPO policy statement on patient information. London: International Alliance of Patients' Organizations, 2009

23. Cox AR, Butt TF. Adverse drug reactions: when the risk becomes a reality for patients. Drug Saf 2012; 35 (11): 977-81

24. Dodoo A, Hugman B. Risk perception and communication in sub-Saharan Africa. Drug Saf 2012; 35 (11): 1041-52

25. Hartigan-Go K. Challenges of drug risk communications in the Philippines. Drug Saf 2012; 35 (11): 995-1004

26. Piening S, Haaijer-Ruskamp FM, de Graeff PA, et al. Healthcare professionals' self-reported experiences and preferences related to direct healthcare professional communications: a survey conducted in the Netherlands. Drug Saf 2012; 35 (11): 1061-72

27. Edwards B, Chakraborty S. Risk communication and the pharmaceutical industry: what is the reality? Drug Saf 2012; 35 (11): 1027-40

28. Fischhoff B. Good decision making requires good communication. Drug Saf 2012; 35 (11): 983-93

29. Larson H, Brocard Paterson P, Erondu N. The globalization of risk and risk perception: why we need a new model of risk communication for vaccines. Drug Saf 2012; 35 (11) $1053-9$

30. Hugman B. Protecting the people? Risk communication and the chequered history and performance of bureaucracy. Drug Saf 2012; 35 (11): 1005-25

31. Uppsala Monitoring Centre. Effective communication in pharmacovigilance: the Erice report (report on the 
International Conference on Developing Effective Communications in Pharmacovigilance held in Erice, 24-27 September 1997, organised by the UMC, the World Health Organization, the University of Verona, the International School of Pharmacology, the Ettore Majorana Centre for Scientific Culture and supported by EQUUS Communications). Uppsala: Uppsala Monitoring Centre, 1997
32. Erice statement 2009: communication, medicines and patient safety. Br J Clin Pharmacol 2010; 69: 207-8

Correspondence: Dr Priya Bahri, European Medicines Agency, 7 Westferry Circus, London, E14 4HB, UK.

E-mail: Priya.Bahri@ema.europa.eu 